\title{
Relação de perdas dentárias e excesso de peso em adultos
}

\author{
Relation of dental losses and excess weight in adults \\ Relación de pérdidas dentales y exceso de peso en adultos
}

Pettra Blanco Lira Matos ${ }^{1}$, Manoel Maria de Souza Benchimol Junior ${ }^{1}$, Naíza Nayla Bandeira de Sá1, Adalberto Lirio de Nazaré Lopes ${ }^{1}$, Dimitra Castelo Branco ${ }^{1}$, Rosana Nazaré Leão Sousa, Cecilia Abrahao Nascimento de Santi ${ }^{1}$, Gabriel Mácola de Almeida ${ }^{1}$, Hélder Antônio Rebelo Pontes ${ }^{1}$, Flávia Sirotheau Correa Pontes ${ }^{1}$, Liliane Silva do Nascimento ${ }^{1}$.

\section{RESUMO}

Objetivo: estudar a relação entre perdas dentárias e o excesso de peso em adultos de Belém do Pará. Métodos: trata-se de um estudo transversal, realizado no período de janeiro a novembro de 2018. Para a avaliação da relação da perda dentária e do excesso de peso dos indivíduos entrevistados, foi utilizado o teste de correlação de Spearman para duas variáveis quantitativas, considerando-se o valor de $p<0,05$. Resultados: participaram 51 adultos usuários do Sistema Único de Saúde da rede municipal, sendo 68,63\% do sexo feminino e $31,37 \%$ do sexo masculino com média de idade de 38,9 anos. A média do Índice de Massa Corpórea foi de $29,7 \mathrm{~kg} / \mathrm{m}^{2}$. O índice CPO-D médio foi 11,68 e a média de dentes perdidos foi de 7,86 . Verificou-se que não houve correlação entre a perda dentária e o excesso de peso, não apresentando correlação entre estas variáveis. Conclusão: a relação nutricional e saúde bucal é fundamental considerar outros fatores associados. Assim a saúde oral não deve ser expressa como um indicador de excesso de peso corporal, sendo necessária a condução de outros estudos.

Palavras chave: Sobrepeso, Obesidade, Perda de dente, Saúde bucal.

\begin{abstract}
Objective: study the relationship between dental losses and excess weight in adults from Belém do Pará. Methods: This is a cross-sectional study carried out between January and November 2018. For the evaluation of the relationship between tooth loss and excess of the individuals interviewed, the Spearman correlation test was used for two quantitative variables, having a significance level of $p<0.05$. Results: Participants were 51 adult users of the Unified Health System of the municipal network, $68.63 \%$ female and $31.37 \%$ male and mean age 38.9 years. The mean Body Mass Index was $29.7 \mathrm{~kg} / \mathrm{m} 2$. The mean CPO-D index was 11.68 and the mean number of teeth lost was 7.86. It was verified that there was no association between dental loss and overweight, with no correlation between these variables. It is noticed that for the nutritional relation and oral health it is fundamental to consider other associated factors. Conclusion: Thus, oral health should not be expressed as an indicator of excess body weight, and other studies should be conducted.
\end{abstract}

key words: Overweight, Obesity, Tooth loss, Oral health.

\section{RESUMEN}

Objetivo: estudiar la relación entre pérdidas dentales y el exceso de peso en adultos de Belém do Pará. Métodos: se trata de un estudio transversal, realizado en el período de enero a noviembre de 2018. Para la evaluación de la relación de la pérdida dental y del peso el exceso de peso de los individuos entrevistados,

1 Universidade Federal do Pará, Belém. *E-mail: profaliliane@ufpa.br 
se utilizó la prueba de correlación de Spearman para dos variables cuantitativas, considerando el valor de $p$ $<0,05$. Resultados: participaron 51 adultos usuarios del Sistema Único de Salud de la red municipal, siendo $68,63 \%$ del sexo femenino y $31,37 \%$ del sexo masculino con promedio de edad de 38,9 años. El promedio del Indice de Masa Corpórea fue de 29,7 kg / m2. El índice CPO-D medio fue 11,68 y el promedio de dientes perdidos fue de 7,86 . Se verificó que no hubo correlación entre la pérdida dental y el exceso de peso, no presentando correlación entre estas variables. Conclusión: la relación nutricional y la salud bucal es fundamental considerar otros factores asociados. Así la salud oral no debe ser expresada como un indicador de exceso de peso corporal, siendo necesaria la conducción de otros estudios.

Palabras clave: Sobrepeso, Obesidad, Pérdida de dientes, Salud bucal.

\section{INTRODUÇÃO}

A perda dentária é resultante de fatores complexos, desencadeada pela precariedade da saúde bucal, por consequência de processos cariosos, doença periodontal, tabagismo e traumatismos (ESPINHA CG, 2016; MOURA SMS et al, 2016). Alterações na saúde sistêmica e diminuição na qualidade de vida podem estar relacionadas com a ingestão inadequada de nutrientes que exige a presença de uma dentição funcional, a presença de pelo menos vinte dentes ao longo da vida (RODRIGUES AMNF, 2014; LIMA CV, et al, 2018). A ausência dos dentes causa comprometimento da capacidade mastigatória levando a limitação do tipo de alimento ingerido e estado nutricional deficiente, pois, interfere na escolha dos alimentos em função de sua consistência e, geralmente, de baixo valor nutricional (YAMASHITA JM, 2013; ARAUJO DS, et al, 2016).

Em edêntulos, o uso de prótese total auxilia na reabilitação da função mastigatória, no entanto, verifica-se que usuários de próteses totais mastigam de 75 a 85\% com menos eficiência em relação aos indivíduos com dentes naturais. Problemas de mastigação e restrição de alimentos podem acometer pessoas com menos de 21 dentes naturais (MOURA SMS, et al., 2016; TSAI S, et al, 2015).

A ausência de elementos dentários e lesões cariosas relacionam-se com o aumento do Índice de Massa Corporal (IMC), a relação entre saúde bucal e obesidade pode corresponder a doenças orais e ao impacto na capacidade mastigatória, marcada por uma dieta desequilibrada, estimulado o crescimento de bactérias cariogênicas (SAIBERT A, et al, 2016). O IMC tem sido o critério de diagnóstico do sobrepeso/obesidade nos serviços de saúde (PILOTTO LM, 2011; DIAS PC, et al, 2017). Para o estudo do excesso de peso é possível recorrer às medidas antropométricas como peso e altura; amplamente utilizadas em função de sua simplicidade, fácil reprodução e baixo custo para determinar a proporção da gordura corporal de cada indivíduo. Nos estudos epidemiológicos em adultos, o sobrepeso e obesidade são definidos diretamente através dos valores do IMC, resultantes da avaliação do peso e altura (COSTA MVC, 2013; ABESO 2016).

A prevalência de sobrepeso e obesidade aumenta de forma preocupante em muitos países, sejam eles desenvolvidos ou em desenvolvimento, e sua população independentemente de gênero, raça, idade e classe social; conduzindo comorbidades (SIQUEIRA DB, et al, 2015; MENDES AA, et al, 2016). A obesidade é uma condição em avanço no mundo e compreende um problema em saúde pública; definida como um acúmulo anormal ou excessivo de gordura corporal e resultando em maiores riscos à saúde, como hipertensão arterial, diabetes tipo 2, osteoartrite, doenças cardiovasculares e alguns tipos de cânceres (BRIANEZZI LFF, et al, 2013; SANTOS TM, 2014; NASCIMENTO GC, 2015).

Poucos estudos têm associado perda dentária e excesso de peso, sendo muitos observados relacionando cárie dentária, doença periodontal e obesidade. Nessa lógica, este estudo objetivou estudar a relação de perdas dentárias e o excesso de peso em adultos de Belém - Pará.

\section{MÉTODOS}

Trata-se de estudo transversal realizado no município de Belém, no período de janeiro a novembro de 2018, em unidades básicas de saúde da área urbana e ribeirinhas. A amostra foi obtida por conveniência, sendo constituída de adultos na faixa etária de 18 a 59 anos, de ambos os sexos. O critério de inclusão foi a 
assinatura do Termo de Compromisso Livre e Esclarecido, adultos com IMC na categoria de excesso de peso, igual ou superior a $25 \mathrm{~km} / \mathrm{m}^{2}$ e com perdas dentárias.

Participaram deste estudo 51 adultos residentes no município de Belém, usuários de seis unidades básicas de saúde, sendo três unidades básicas de saúde da área urbana (Jurunas, Guamá e Condor) que foram sorteadas aleatoriamente e de três UBS dos povos das águas (Genipaúba, Aurá e São Pedro) que foram selecionadas pelo Programa Luz na Amazônia da Universidade Federal do Pará.

Coletaram-se informações sociodemográficas, hábitos de higiene e história odontológica por meio de aplicação de instrumento validado. As variáveis incluídas foram idade, sexo, renda e escolaridade; e ainda prática de escovação diária, uso de fio dental e visita ao dentista. Realizou-se exames clínicos individuais de acordo com os critérios adotados no projeto SBBrasil (BRASIL, 2009), conduzidos por dois examinadores calibrados para aferição dos índices de condição dentária (CPO-D).

A avaliação ocorreu sob luz natural em condições ambientes com auxílio de espelho clínico plano, espátulas de madeiras e equipamentos de proteção individual. O índice CPO-D calcula a somatória de dentes permanentes cariados $(C)$, perdidos $(P)$ e obturados $(O)$; em relação ao número de indivíduos examinados em uma determinada população. É considerado de fácil aplicação e confiável (MOURA SMS, et al, 2016; MONTEIRO IIS, 2015)

Para a avaliação antropométrica foram seguidas as orientações do Sistema de Vigilância Alimentar e Nutricional. Na aferição do peso utilizou-se balança plataforma mecânica, confeccionada de material resistente com cursores de aço inoxidável polido e capacidade de pesagem de $200 \mathrm{~kg}$, a qual é empregada no serviço da UBS; com o indivíduo posicionado de costas para a balança e descalço no centro desta, ereto e com os pés juntos e braços estendidos ao longo do corpo. Para a aferição da altura, utilizou-se antropômetro com escala até 2,00 metros, um dispositivo fixado na coluna de sustentação da balança; no qual a aferição foi realizada com o indivíduo ereto, de pé, no centro do equipamento com a cabeça livre de adereços, erguida e olhando para um ponto fixo e com os braços estendidos ao longo do corpo.

Para a população brasileira, o diagnóstico do estado nutricional utiliza a recomendação proposta pela Organização Mundial em Saúde para classificação de sobrepeso e obesidade; assim faixas pré-determinadas apontam o padrão corpóreo do indivíduo adulto, estipulando denominar de sobrepeso o IMC de 25 a $29 \mathrm{~kg} / \mathrm{m}^{2}$ e obesidade o IMC $\geq 30 \mathrm{~kg} / \mathrm{m}^{2}$ e de excesso de peso o IMC $\geq 25 \mathrm{~kg} / \mathrm{m}^{2}$ (incluindo a obesidade). Nessa classificação ainda há os pontos de corte de IMC $<18,5 \mathrm{~kg} / \mathrm{m}^{2}$ (baixo peso) e IMC $\geq 18,5$ e $<25 \mathrm{~kg} / \mathrm{m}^{2}$ (adequado ou eutrófico). O IMC é um dos índices antropométricos mais aplicados para apurar a disposição nutricional, relacionando o peso pela estatura ao quadrado (ABESO, 2016; NASCIMENTO GC; 2015; SILVA $A R, 2014)$.

As variáveis consideradas neste estudo foram a ocorrência de perdas dentárias obtidas a partir dos códigos 4 e 5 do índice CPO-D, que representam os dentes permanentes perdidos por cárie e por outras razões, respectivamente; e o excesso de peso, obtido pelo IMC. Os dados coletados foram tabulados no Microsoft Excel 2016 e analisados no software BioEstat 5.0. Para a avaliação da relação da perda dentária e do excesso de peso dos indivíduos entrevistados, utilizou-se o teste de correlação de Spearman para duas variáveis quantitativas, adotando-se $o$ valor de $p \leq 0,05$. Considerou-se como hipótese nula que a perda dentária não está associada ao excesso de peso; e como hipótese alternativa que a perda dentária está associada ao excesso de peso. Esta pesquisa foi aprovada pelo Comitê de Ética em Pesquisa envolvendo seres humanos do Instituto de Ciências da Saúde da Universidade Federal do Pará, seguindo as normas da Resolução n 466/12 do Conselho Nacional de Saúde sob o parecer nº 877.309.

\section{RESULTADOS E DISCUSSÃO}

Observou-se que $68,63 \%(n=35)$ eram do sexo feminino e $31,37 \%(n=16)$ do sexo masculino. Dessa amostra a média de idade foi de 38,9 anos. A renda familiar mensal de $66,67 \%(n=34)$ dos usuários é de até um salário mínimo, de $21,57 \%(n=11)$ até dois salários mínimos e $11,76 \%(n=6)$ de dois até quatro salários mínimos; quanto à escolaridade $32,94 \%(n=27)$ possui ensino fundamental incompleto e $19,6 \%(n=10)$ possui 
ensino médio completo, apenas $1,96 \%(n=1)$ possui ensino superior completo. $E$ referência à profissão, $35,29 \%(n=28)$ são pescadores extrativistas, sendo a maioria da amostra, e $21,56 \%$ ( $n=11)$ são donas de casa (Tabela 1).

Tabela 1 - Representação dos dados sociodemográficos dos participantes, Pará, 2018.

\begin{tabular}{llcc}
\hline Variável & Resultados & $\mathbf{n}$ & $\%$ \\
\hline \multirow{2}{*}{ Sexo } & Feminino & 35 & 68,63 \\
& Masculino & 16 & 31,37 \\
\hline \multirow{2}{*}{ Renda familiar mensal } & Até 1 salário mínimo & 34 & 66,67 \\
& Até 2 salário mínimo & 11 & 21,57 \\
& De 2 a 4 salários mínimos & 6 & 11,76 \\
\hline \multirow{3}{*}{ Escolaridade } & Fundamental incompleto. & 13 & 45,50 \\
& Fundamental completo & 27 & 32,94 \\
& Ensino médio & 10 & 19,60 \\
\hline \multirow{2}{*}{ Profissão } & Superior & 1 & 1,96 \\
& Pescadores & 28 & 35,29 \\
& Dona de casa & 11 & 21,56 \\
\hline
\end{tabular}

Fonte: Matos PBL, et al., 2019.

Com relação aos hábitos de higiene verificou-se que 92,16\% $(n=47)$ dos usuários entrevistados já tiveram consulta odontológica, no entanto 7,85\% $(n=04)$ nunca foram ao dentista; $37,25 \%(n=19)$ deles realizaram sua última consulta em um período superior a dois anos e 19,61\% $(n=10)$ em um intervalo de um a dois anos. Quanto à frequência de escovações diárias, $64,70 \%(n=33)$ afirmaram escovar os dentes três vezes ao dia, $27,45 \%(n=14)$ duas escovações ao dia e 5,88\% ( $n=3)$ apenas uma vez ao dia. O uso diário de fio dental foi declarado por $49 \%(n=25)$ dos entrevistados, porém $51 \%(n=26)$ deles não apresentam o hábito de utilizar fio dental; ainda foi revelado que $5,88 \%(n=3)$ fazem compartilhamento de escova dental.

Tabela 2 - Representação dos dados referentes ao cuidado em saúde bucal, Pará, 2018.

\begin{tabular}{llcc}
\hline Variável & Resultados & $\mathbf{n}$ & $\%$ \\
\hline \multirow{2}{*}{ Já foi ao dentista } & Sim & 47 & 92,16 \\
& Não & 4 & 7,84 \\
\hline \multirow{2}{*}{ Quando foi a última consulta ao dentista } & Há mais de dois anos & 19 & 37,25 \\
& Há menos de dois anos & 10 & 19,61 \\
& Há menos de um ano & 18 & 35,30 \\
& Nunca & 4 & 7,84 \\
\hline \multirow{2}{*}{ Frequência de escovação } & $3 x /$ dia & 33 & 64,70 \\
& $2 x /$ dia & 14 & 27,45 \\
\multirow{2}{*}{ Usa fio dental } & $1 x /$ dia & 5 & 5,88 \\
\hline \multirow{2}{*}{ Escova compartilhada } & Sim & 25 & 49,00 \\
& Não & 26 & 51,00 \\
\hline
\end{tabular}

Fonte: Matos PBL, et al., 2019.

A média do IMC dos indivíduos foi de $29,7 \mathrm{~kg} / \mathrm{m}^{2}$ e sua análise evidenciou que $66,67 \%$ ( $\left.\mathrm{n}=34\right)$ dos indivíduos estavam na faixa de sobrepeso, enquanto $33,33 \%(n=17)$ são classificados na faixa de obesidade; representando a amostra com excesso de peso.

$\mathrm{Na}$ avaliação odontológica verificou-se $26,85 \%(n=160)$ dentes cariados, $67,28 \%(n=401)$ dentes perdidos e 5,87 ( $n=35)$ dentes obturados; onde a média de dentes perdidos foi de 7,86 e o índice CPO-D médio foi de 
11,68. Observou-se que não há associação estatística entre a perda dentária e o excesso de peso ( $\mathrm{p}=0,2667)$, assim como não se observou correlação entre a perda dentária e o excesso de peso nesta amostra ( $r s=$ 0,1585).

Não houve correlação entre perda dentária e excesso de peso. A perda de elementos dentários, principalmente posteriores e antagonistas, o não uso de próteses dentárias ou a sua má adaptação influenciam na eficácia mastigatória, que pode não gerar um estado nutricional deficitário, mas afeta a escolha dos alimentos e reflete a qualidade de vida (POEIRAS C, 2013; NEVES VF, 2013).

Um estudo de revisão buscou observar a existência de uma possível relação entre a obesidade e as doenças bucais mais comuns e que ocasionam a perda dentária; onde os resultados se mostraram controversos, existindo falta de evidências que suportem essa associação (SAPORITI JM, et al, 2014).

A associação da má condição oral, representada pela perda dos dentes, com o baixo peso ou excesso de peso, através do IMC, realizada em um estudo de 900 idosos independentes; demonstrou que os indivíduos edêntulos que não utilizavam próteses dentárias tinham maior probabilidade de estar não apenas com sobrepeso/obesidade, mas também abaixo do peso (TORRES LHN, 2013).

A relação entre saúde bucal e o estado nutricional entre idosos, onde a maioria se encontrava edêntulo e com déficit nutricional. Foram verificadas correlações entre parâmetros estabelecidos, dentre os quais se avaliou o CPO-D versus IMC, que demonstrou uma correlação fraca; os parâmetros como pH da saliva, capacidade tampão e fluxo salivar também foram avaliados e ainda não apresentam relação bem esclarecida. Assim, concluiu-se que a saúde bucal e o estado nutricional estão inter-relacionados, porém com dados insuficientes para estabelecer a natureza da relação (BEZERRA GS, et al, 2015).

A relação cárie dentária e obesidade entre crianças e adolescentes em diferentes continentes, foi investigada em uma revisão sistemática de 31 artigos publicados entre 2006 e 2016; onde a maioria dos artigos selecionados não demonstrou associação entre obesidade e cárie dentária em crianças e adolescentes em todos continentes, com exceção da América do Norte e Oceania BATTAGLIA G, 2017)

A avaliação da associação entre perda dentária e sobrepeso/obesidade em uma população adulta brasileira foi realizada em um estudo transversal com adultos obesos e quase todos ou todos os dentes ausentes, por meio de regressões múltiplas ordinais e após ajuste dos fatores de confusão; observando que sobrepeso/obesidade não apresentam associações estatísticas com perdas dentárias, mas sim fatores de risco comuns (PILOTTO JM, et al, 2014). A associação entre perda dentária e obesidade entre idosos foi analisada por um estudo prospectivo de coorte, na qual modelos de regressão logística multivariada foram utilizados para esta avaliação; onde não foi observada qualquer associação entre obesidade geral e perda dentária (SINGH A, et al, 2015).

A avaliação odontológica deste estudo demonstrou a perda dentária com a maior prevalência em relação aos demais componentes do índice CPO-D, corroborando com os resultados do último inquérito nacional SBBrasil 2010 (BRASIL, 2009), que demonstrou perda dentária extremamente elevada entre adultos e idosos (CHAVES SCL, et al, 2017).

O estudo constatou que a grande maioria dos entrevistados apresenta renda mensal de até um salário mínimo; sendo esta condição socioeconômica baixa relacionada ao aumento da incidência de obesidade e explicada na literatura, pelo acesso aos alimentos industrializados de alto valor calórico e de baixo custo (PASSERI CR, 2015).

O rendimento ainda afeta a saúde bucal do indivíduo, pois a baixa renda ocasiona dificuldades de acesso a tratamentos e serviços e pouca informação em saúde (BORGES TS, et al, 2016); podendo ser observado com os dados obtidos dos hábitos de higiene oral, onde 5,88\% escovam seus dentes apenas uma vez ao dia, $5,88 \%$ fazem compartilhamento de escova e 7,85\% da amostra deste estudo nunca foram ao dentista. Este último concorda com a Pesquisa Nacional por Amostra de Domicílio realizada em 2008; que investigou a utilização de serviços odontológicos e verificou que $11,7 \%$ da população brasileira não havia ido ao dentista (CHAVES SCL, et al, 2017). 


\section{CONCLUSÃO}

A observação das condições de saúde bucal e do IMC dos usuários do município de Belém não revelou correlação entre a perda dentária e o excesso de peso. Destaca-se que a relação nutricional e saúde bucal ainda é controversa, a saúde oral não pode ser interpretada como um indicador de excesso de peso corporal. É fundamental considerar outros fatores associados e a condução de novos estudos buscando mais conhecimentos sobre esta relação. No entanto, o fortalecimento das políticas de saúde para preservação dos elementos dentários naturais pode ter impacto positivo no estado nutricional e consequentes morbidades. Assim, é necessária a conscientização multiprofissional na saúde para uma condição bucal satisfatória e manutenção do estado nutricional adequado dos usuários.

\section{AGRADECIMENTOS E FINANCIAMENTO}

Agradecemos a Universidade Federal do Pará e a Secretaria Municipal de Saúde de Belém pelo apoio na realização da pesquisa.

\section{REFERÊNCIAS}

1. ARAUJO DS, MARQUEZIN MCS, BARBOSA TS, GAVIAO MBD, CASTELO PM. Evaluation of masticatory parameters in over weight and obese children. European Journal of orthodontics. 2016: 1(1): 393-397.

2. ASSOCIAÇÃO BRASILEIRA PARA O ESTUDO DA OBESIDADE E SÍNDROME METABÓLICA. Diretrizes Brasileiras de Obesidade. $4^{\circ}$ ed. São Paulo: ABESO; 2016.

3. BATTAGLIA G. Cárie dentária e obesidade em crianças e adolescentes em diferentes continentes: revisão sistemática [Dissertação]. Piracicaba: Universidade Estadual de Campinas; 2017.

4. BEZERRA GS, NETA MVB, LOPES RGM, MOURA IS, SANTANA MDR, ABREU LC. Relação entre saúde bucal e o estado nutricional em idosos. Revista e-ciência. 2015; 3(1): 15-21.

5. BORGES TS, RENTER CP, SCHWANKE NL, NETO LK, GRAZZIOTIN GB, BURGOS MS. Relação entre obesidade e presença de cárie dentária em adolescentes no município Santa Cruz do Sul - RS, Brasil. Adolesc. Saúde. 2016; 13(4): 25-32.

6. BRASIL. Ministério da Saúde. Secretaria de vigilância em Saúde. Departamento de atenção básica. SBBrasil 2010: manual da equipe de campo. Brasília; 2009.

7. BRIANEZZI LFF, AL-AHJ LP, PRESTES LA, ANDREATTA LM, VASCONCELOS LRM, MARSICANO JÁ. Impacto da obesidade na saúde bucal: revisão de literatura. RFO. 2013; 18(2): 211-216.

8. CHAVES SCL, ALMEIDA AMFL, ROSSI TRA, SANTANA SF, BARROS SG, SANTOS CML. Política de saúde bucal no Brasil 2003-2014: cenário, propostas, ações e resultados. Ciência \& Saúde Coletiva. 2017; 22(6): 1791-1803.

9. COSTA MVC. Associação entre índice de massa corporal e condições de saúde bucal de adolescentes de CuiabáMT [Dissertação]. Cuiabá: Universidade de Cuiabá; 2013.

10. DIAS PC, HENRIQUE P, ANJOS LA, BURLANDY L. Obesidade e políticas públicas: concepções e estratégias adotadas pelo governo brasileiro. Cad. Saúde Pública. 2017; 33 (7):

11. ESPINHA CG. A influência do edentulismo no declínio das capacidades cognitivas [tese]. Porto: Universidade do Porto; 2016.

12. LIMA CV, SOUZA JGS, OLIVEIRA BEC, NORONHA MN, PEREIRA AC, PRPBST LV. Falta de dentição funcional influencia na autopercepção da necessidade e tratamento em adultos: estudo de base populacional no Brasil. Cad. Saúde Colet. 2018; 26 (1): $63-69$.

13. MENDES AA, IEKER ASD, CASTRO TF, AVELAR A, NARDO JÚNIOR N. Multidisciplinar programs for obesitytreatment in Brazil: a systematicreview. Rev. Nutr. 2016; 29(6): 867-884.

14. MONTEIRO IIS. A relação entre a prevalência de cárie dentária e o índice de massa corporal em pacientes da clínica dentária Egas Moniz na primeira consulta de triagem [tese]. Portugal: Instituto superior de Ciências da Saúde Egas Muniz; 2015.

15. MOURA SMS, LEITE CMC, ROCHA AMC, COSTA MAB, OLIVEIRA IM, CARDOSO JC. Relação entre nutrição de idosos e dentição: revisão de literatura. Jorn. Inter. Bioc. 2016; 1(1): 5-8.

16. NASCIMENTO GG. Impacto de sobrepeso e obesidade ao longo da vida na ocorrência de doença periodontal: evidências de revisões sistemáticas e de estudos transversais e longitudinais [tese]. Pelotas: Universidade Federal de Pelotas; 2015. 
17. NEVES VF. Saúde oral e nutrição como promotores de qualidade de vida no idoso [Dissertação]. Porto: Universidade Fernando Pessoa; 2013.

18. PASSERI CR. Epidemiologia e parâmetros laboratoriais relacionados à perda dentária em pacientes obesos candidatos a cirurgia bariátrica [Dissertação]. Bauru: Universidade de são Paulo; 2015.

19. PILOTTO JM, CELESTE RK, FASUSTEIN E, SLAVUTZKY SMB. Association between tooth loss and overweight/obesity among Brazilian adults: the pro-saude study. Braz Oral Res. 2014; 28 (1): 1-6.

20. PILOTTO LM. Perda dental e sua associação com a obesidade em uma população adulta no Brasil [Dissertação]. Porto Alegre: Universidade Federal do Rio Grande do Sul; 2011.

21. POEIRAS C. Importância do estado de saúde oral na nutrição de idosos [Dissertação]. Portugal: Instituto Superior de Ciências da Saúde Egas Moniz; 2013.

22. RODRIGUES AMNF. Eficácia mastigatória em pacientes portadores de prótese parcial removível e prótese parcial fixa - antes e depois [Dissertação]. Viseu: Universidade Católica Portuguesa; 2014.

23. SAIBERT A, FIORI R, LORENA OJ, MARQUES FR, PORTUGAL MEG, DALLEDONE M. Obesidade na adolescência e suas implicações na saúde bucal. Revista Gestão \& Saúde. 2016.15(2): 35-40.

24. SANTOS TM. Perfil da condição bucal de indivíduos obesos [tese]. Salvador: Universidade Federal da Bahia; 2014.

25. SAPORITI JM, VERA BSB, ARRUDA BS, CALDEIRA VS, PEREIRA LGA, NASCIMENTO GG. Obesidade e saúde bucal: impacto da obesidade sobre condições bucais. RFO. 2014; 19 (3): 368-374.

26. SILVA AR. Obesidade e cárie dentária. Coorte de nascimentos de 1993, Pelotas-RS. Pelotas: Universidade Federal de Pelotas; 2014.

27. SINGH A, PERES MA, PERES KG, BERNADO CO, XAVIER A, D'ORSI E. Gender differences in the association between tooth loss and obesity among older adults in Brasil. Rev Saúde Publica. 2015; $49: 44$.

28. SIQUEIRA DB, SOUZA RKT, MESAS AE, SANTOS HG, BORTOLETTO MSS. Diferenças entre sexo nos determinantes da obesidade abdominal em adultos de 40 anos ou mais: estudo de base populacional. Rev. Nutr. 2015; 28(5): 485-496.

29. TORRES LHN, SILVA DD, NERI AL, HILGERT JB, HUGO FN, SOUSA MLR. Association between underweight and overweight/obesity with oral health among independently living Brazilian elderly. Nutrition. 2013; 29(1): $152-157$.

30. TSAI S, LIN M, CHIN W, JANE S, TU L, CHEN M. Factors associated with having less than 20 teeth in rural adults: a cross-sectional study. BMC Oral Heath. 2015; 15:158.

31. YAMASHITA JM. Estudo transversal sobre problemas bucais em pacientes obesos mórbidos [tese]. Bauru: Universidade de São Paulo: 2013. 